\title{
Left Atrial Myxoma Causing Ping-Pong Mitral Stenosis
}

Kunal Mahajan*, Sachin Sondhi, Shivani Rao, Naresh Gaur and Sanjay Rathour

Department of Cardiology, Indira Gandhi Medical College, Shimla, Himachal Pradesh, India

\section{Clinical Image}

A 35-years-old female presented to us for evaluation of a recent episode of syncope. She also complained of exertional breathlessness for past 6 months. She denied experiencing orthopnea, paroxysmal nocturnal dyspnea, fever, weight loss and chest pain. At presentation, her vitals were stable (pulse $=78 / \mathrm{min}$ and regular, $\mathrm{BP}=120 / 78 \mathrm{~mm} \mathrm{Hg}$ ). Cardiac auscultation revealed a mild diastolic murmur at the apex raising possibility of mitral stenosis. On transthoracic echocardiogram, a large $(45 \mathrm{~mm} \times 25 \mathrm{~mm})$ mobile, irregular mass was seen in the left atrium (Figure 1). This mass was hetero-echoic in echotexture and was attached to the inter-atrial septum in the region of fossa ovalis. All these findings were suggestive of the diagnosis of atrial myxoma. It was prolapsing into the left ventricle during diastole (Figure 2). Colour Doppler revealed increased velocity of blood flow across the mitral valve. On continuous-wave Doppler, peak and mean gradients across mitral valve were $18 \mathrm{~mm} \mathrm{Hg}$ and $8 \mathrm{~mm} \mathrm{Hg}$ respectively. However mitral valve was normal in morphology with normal sub-valvular apparatus. All other cardiac valves and chambers were also normal. Patient underwent complete surgical excision of the cardiac mass. Histopathology confirmed it to be a myxoma.

Myxoma is the most frequent primary cardiac tumour accounting for $80 \%$ of all cases [1]. It may originate in any heart chamber, most commonly in the left atrium (75\%) followed by right atrium (23\%) and ventricles (2\%) [2]. Myxoma can be easily misdiagnosed and is initially suspected in only $5.7 \%$ of patients [3]. Depending on the location of myxoma and its association with the heart structures, it can cause clinical manifestations, which include obstructive, embolic and constitutional symptoms [4]. Left atrial myxoma, especially if mobile and large, may result in symptoms similar to mitral stenosis due to obstruction of blood flow across the mitral valve. These symptoms include exertional dyspnea, paroxysmal nocturnal dyspnea, orthopnea, fatigue, and syncope [3]. In conclusion, left atrial myxoma should always be considered in the differential diagnosis in patients with

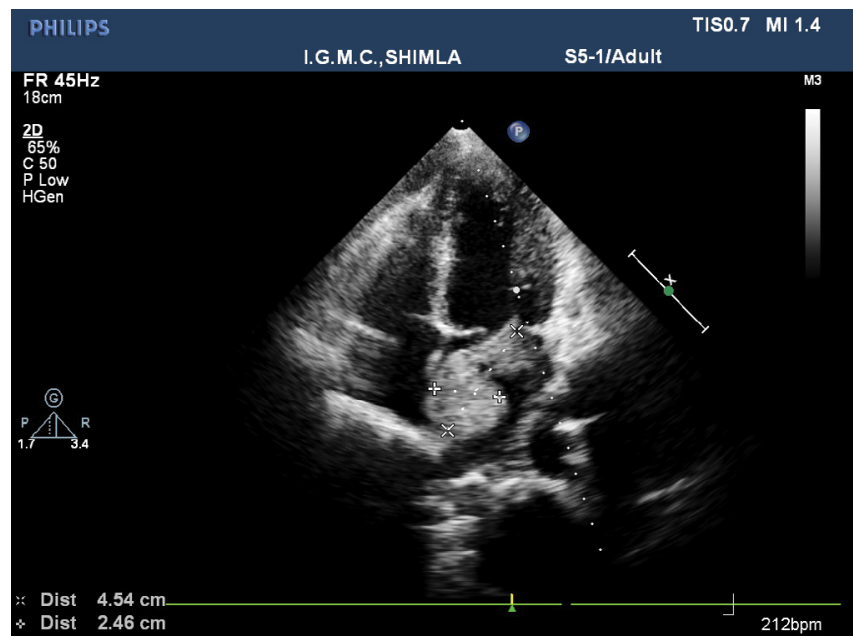

Figure 1: Transthoracic echocardiogram, apical-4-chamber view, showing a large mass in the left atrium with multiple echolucent densities interspersed in an echogenic background.

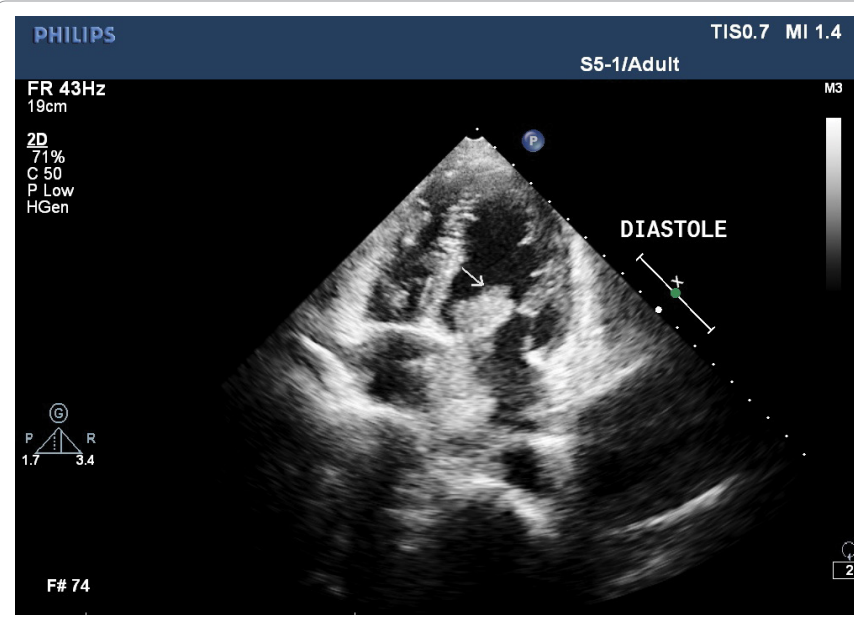

Figure 2: Left atrial myxoma prolapsing into the left ventricle (arrow) through mitral valve causing functional mitral stenosis.

suspected mitral valve disease. Echocardiography should be performed as early as possible to establish a prompt diagnosis and management.

\section{Patient Consent}

Obtained.

\section{Competing interest}

None.

\section{Acknowledgement}

None.

\section{Contributorship statement}

KM made the diagnosis. SS, SR, NG and SaR performed the investigations and were involved in the management of the patient. KM wrote the manuscript and performed the literature search. All authors read and approved the final version of the manuscript.

\section{References}

1. Blondeau P (1990) Primary cardiac tumors-French studies of 533 cases Thorac Cardiovasc Surg 38: 192-195.

2. Diaz A, Di Salvo C, Lawrence D, Hayward M (2011) Left atrial and righ ventricular myxoma: an uncommon presentation of a rare tumour. Interact Cardiovasc Thorac Surg 12: 622-623.

*Corresponding author: Kunal Mahajan, Department of Cardiology, Indira Gandhi Medical College, Shimla, Himachal Pradesh, India, Tel: + 8628864820; E-mail: Kunalmahajan442@gmail.com

Received October 19, 2017; Accepted October 19, 2017; Published October 19 2017

Citation: Mahajan K, Sondhi S, Rao S, Gaur N, Rathour S (2017) Left Atrial Myxoma Causing Ping-Pong Mitral Stenosis. J Cardiovasc Dis Diagn 5: i108. doi: 10.4172/2329-9517.1000i1087

Copyright: (c) 2017 Mahajan K, et al. This is an open-access article distributed under the terms of the Creative Commons Attribution License, which permits unrestricted use, distribution, and reproduction in any medium, provided the original author and source are credited. 
Citation: Mahajan K, Sondhi S, Rao S, Gaur N, Rathour S (2017) Left Atrial Myxoma Causing Ping-Pong Mitral Stenosis. J Cardiovasc Dis Diagn 5 : i108. doi: 10.4172/2329-9517.1000i108

Page 2 of 2

3. Goswami KC, Shrivastava S, Bahl VK, Saxena A, Manchanda SC, et al. (1998) Cardiac myxomas: Clinical and echocardiographic profile. Int J Cardiol 63: 251-259.
4. Pinede L, Duhaut $P$, Loire $R$ (2001) Clinical presentation of left atrial cardiac myxoma. A series of 112 consecutive cases. Medicine 80: 159-172. 